\title{
Aplicaciones de la hidroxiapatita coraíina HAP-200 como material de implante óseo en ortopedia
}

\author{
Pereda O. ${ }^{1}$, González R. ${ }^{2}$
}

\begin{abstract}
RESUMEN
Las hidroxiapatitas coralinas desarrolladas en Cuba han sido evaluadas fundamentalmente en estomatología, pero, no se contaba hasta el momento con un estudio sobre su comportamiento para el tratamiento de defectos óseos de los huesos largos y en șitios de mayor carga mecánica como es el caso de las que comúnmente se tratan en ortopedia.

En el presente trabajo se presenta una parte de las experiencias clínicas que se vienen realizando en el Hospital Carlos J. Finlay con el uso de la hidroxiapatia porosa coralina HAP-200 en ortopedia y traumatología, donde se recogen los resultados obtenidos en 10 pacientes que presentaron lesiones óseas de origen tumoral con diferentes características y magnitud. La evolución clínica, radiográfica y hematológica confirma las excelentes cualidades de este biomaterial para la regeneración del tejico óseo y demuestra sus posibilidades incluso para el tratamiento de grandes defectos.
\end{abstract}

\section{SUMMARY}

The coralina hydroxyapatites developed in Cuba have been evaluated in odontology, but a study of their use in orthopedic cases has not been carried out until now.

The present work shows the results of the use of porous hydroyapatite coralina HAP200 in traumatology and orthopaedics. It includes 10 patients with different tumoral bone diseases which were treated at C.J. Finlay Hospital.

The radiographic, haematological and ciinical evolution confirmed the excellent properties of this biomaterial for the regeneration of bone tissue and suggests its possible use in the treatment of extensive bone defects.

La necesidad de restaurar diferentes tipos de defectos de sustancia ósea ha constituido desde hace mucho un importante tema de estudio e investigación para las ciencias médicas.

Este problema, hasta hace muy poco, sólo había encontrado solución parcial en los injertos de huesohomólogoo heterólogoyenlos deslizamientos

1 Doctor en Medicina, Servicio de Ortopedia, Hospital. C. J. Finlay, La. Habana, Cuba

2 Doctor en Ciencias Químicas, Investigador, Laboratorio. Biomateriales, CNIC, Apdo 6880, La Habana, Cuba o transportación de fragmentos óseos, con las consiguientes dificultades de incorporación, riesgos quirúrgicos y complejidades de la técnica.

En el presente siglo, muchos investigadores se han dado a la tarea de obtener materiales biocompatibles para la sustitución del hueso, lo cual ha sido posible gracias al avance actual de la ciencia de los materiales, la química y la tecnología médica mundial (1-5). Hoy es posible disponer de un grupo de cerámicas implantológicas de amplia utilización por su extraordinaria identificación química con las estructuras del hueso 
y condiciones de biocompatibilidad necesarias para la inserción dentro del organismo humano. Dentro de este grupo, la hidroxiapatita es uno de los biomateriales más bioactivo y biocompatible que se conoce y es reconocido en la actualidad como uno de los más perspectivos para su uso en la implantología ósea, tanto por sus propiedades intrínsecas como por la posibilidad de su síntesis por medios industriales (6-13).

La hidroxiapatita porosa coralina HAP-200 se produce en Cuba a partir de corales marinos y ha sido sometida a pruebas clínicas controladas en animales, realizándose estudios clínicos, histopatológicos y cultivos de tejido in vitro que validan la biocompatibilidad del material y garantizan su aplicabilidad en la práctica médica (14).

En general, los biomateriales de fosfatos de calcio e hidroxiapatita han sido empleados ampliamente en cirugía endobucal y maxilofacial con excelentes resultados (15-26). Sin embargo, su uso no ha sido muy extendido en ortopedia y traumatología, especialidades a las cuales la vida moderna imprime cada vez más exigencias dada la frecuencia y magnitud de los accidentes, responsables directos de grandes pérdidas de sustancia ósea, así como por otras causas, a las que los biomateriales pueden ofrecer una feliz solución.

En este trabajo, se reportan las experiencias y los resultados obtenidos en la práctica clínica en ortopedia y traumatología, con el uso de la coralina HAP-200 como biomaterial de relleno en defectos de sustancia ósea de diferente naturaleza en esta especialidad.

\section{Materiales y métodos}

En el presente reporte se incluye sólo una muestra de 10 pacientes de los tratados en el servicio de ortopedia del Hospital C.J. Finlay de La Habana, como parte del protocolo de investigación y evaluación clínica de los biomateriales coralina, que son representativos de algunos tipos de lesiones de difícil solución y que han sido tratadas con este biomaterial.

Todos los pacientes fueron sometidos a cuidadosos exámenes clínicos, radiográficos e histológicos que permitieron diagnosticar con certeza que presentaban lesiones óseas tumorales benignas de la pelvis o las extremidades, las cuales fueron clasificadas por grados según el tamaño de las mismas (A:menores de $2 \mathrm{~cm}$; $B$ : de 2 a $5 \mathrm{~cm}$; C: de 5 a $10 \mathrm{~cm}$ y D: mayores de $10 \mathrm{~cm}$ ) (tabla 1). No hacemos mención aparte al sexo y la raza, por cuanto no tienen valor significativo en relación a su patología o tratamiento. Ningún paciente presentaba enfermedades sistémicas y en los casos de sepsis, éstas fueron tratadas previamente.

El método de tratamiento consistió en el curetaje quirúrgico o exéresis de la lesión tumoral, lavado cuidadoso del lecho receptor y relleno del defecto óseo resultante con biomaterial coralina HAP200 , extremando las medidas de asepsia en la manipulación e implante de la cerámica y observando las indicaciones para esta técnica (27).

El biomaterial usado fue hidroxiapatita HAP-200 (figura 1) en forma de granulado de tamaño grande $(2-2,5 \mathrm{~mm})$ y en cantidad suficiente para el relleno del defecto óseo.

El seguimiento clínico se realizó por observación directa del paciente, control de los signos vitales y estudio local de la herida quirúrgica, mediante una clasificación en grados (desde 0 hasta III) para la evaluación clínica del edema, dolor, inflamación, sepsis o exposición del material, así como por estudios radiográficos.

El seguimiento radiográfico se hizo a través de radiografías simples a los 30,90 y 180 días, y luego cada 6 meses. Las imágenes radiográficas permitieron evaluar el comportamiento del implante y la interfase hueso-implante.

También se realizó el estudio comparativo de la densidad ósea relativa del implante mediante sistema de procesamiento digital de imágenes Ortopack [28].

La evaluación hematológica se hizo mediante el estudio del hemograma completo, eritrosedimentación, calcio, fósforo y fosfatasa alcalina en los períodos de tiempo ya señalados. 
Tabla 1
No.
Edad
Diagnóstico
Tiempo evolución
Tipo
Grado
(Postoperatorio)

\begin{tabular}{|c|c|c|c|c|}
\hline 1 & 23 & $\begin{array}{l}\text { Quiste óseo solitario recidivante } \\
\text { en fémur izquierdo }\end{array}$ & B & 32 meses \\
\hline 2 & 18 & $\begin{array}{l}\text { Defecto fibroso metafisario } \\
\text { recidivante, tibia izquierda }\end{array}$ & B & 18 meses \\
\hline 3 & 31 & $\begin{array}{l}\text { Quiste óseo simple de } \\
\text { pelvis }\end{array}$ & A & 16 meses \\
\hline 4 & 35 & $\begin{array}{l}\text { Osteomielitis crónica focal, } \\
\text { tibia izquierda }\end{array}$ & A & 16 meses \\
\hline 5 & 22 & $\begin{array}{l}\text { Condroma calcáneo } \\
\text { derecho }\end{array}$ & A & 16 meses \\
\hline 6 & 18 & $\begin{array}{l}\text { Condroblastoma fémur } \\
\text { izquierdo }\end{array}$ & $\mathrm{C}$ & 12 meses \\
\hline 7 & 19 & $\begin{array}{l}\text { Quiste óseo simple, húmero } \\
\text { izquierdo }\end{array}$ & B & 9 meses \\
\hline 8 & 58 & $\begin{array}{l}\text { Quiste óseo, radio } \\
\text { derecho }\end{array}$ & B & 13 meses \\
\hline 9 & 18 & $\begin{array}{l}\text { Defecto fibroso metafisario } \\
\text { distal }\end{array}$ & B & 12 meses \\
\hline 10 & 26 & $\begin{array}{l}\text { Quiste óseo, quinto metatarsiano } \\
\text { derecho }\end{array}$ & A & 12 meses \\
\hline
\end{tabular}

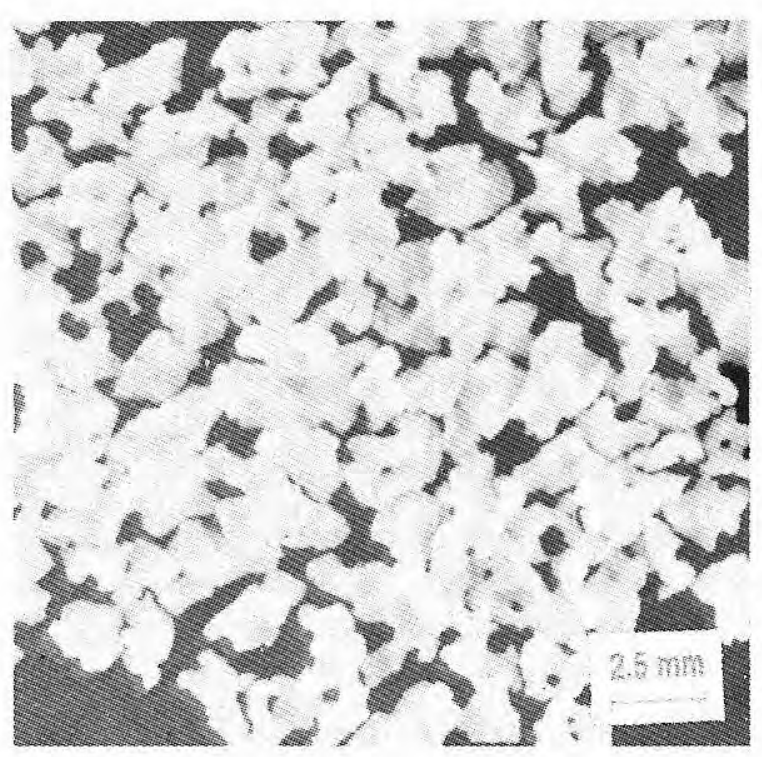

figura 1.-Hidroxiapatita porosa coralina HAP-200 en forma de granulados, con poros entre 5 y $340 \mathrm{~mm}$ y tamaño de las partículas de 2-2,5 $\mathrm{mm}$.

\section{Resultados y discusión}

Uno de los aspectos señalados como críticos en el empleo de biomateriales en general y de los diferentes tipos de hidroxiapatitas en particular, en forma de granulados, está relacionado con la posible exfoliación o migración de las partículas, por lo que fue tenido en cuenta y se tomaron las precauciones para evitarlo en el acto quirúrgico. No obstante, observamos que se produjo expulsión de unos pocos gránulos aislados en uno de los pacientes, probablemente por ligero sobrerrelleno del defecto. La expulsión ocurrió en el primer mes de evolución y por la propia herida quirúrgica. Después de ello, la herida sanó por completo y actualmente es el paciente de mayor tiempo de evolución en este grupo.

Todos los pacientes presentaron una evolución normal en el sitio quirúrgico y una respuesta clínica satisfactoria según los resultados de los análisis de control. 
$\mathrm{Ni}$ el dolor ni el edema mostraron variaciones significativas relacionadas con el implante o diferentes de la evolución postoperatoria habitual. No aparecieron signos flogísticos locales, ni sepsis en ninguno de los casos (tabla 2).

Los parámetros hematológicos, así como los niveles de calcio y fósforo se mantuvieron normales durante todo el período de evolución (figura 2).

La eritrosedimentación se encontró elevada en el preoperatorio de la paciente con condroblastoma de fémur, normalizándose la misma en el curso del primer mes de postoperatorio.

La fosfatasa alcalina estuvo elevada desde el preoperatorio en dos pacientes con diagnóstico de condroblastoma de fémur y defecto fibroso metafisario de tibia, respectivamente. Esto encuentra explicación en la actividad osteo y fibroblástica de estos tumores [29]. Después del primer mes de evolución la fosfatasa volvió a sus valores normales (figura 2).

Es de destacar que en el resto de los pacientes ocurrió un discreto aumento de la fosfatasa alca- lina en el primer mes de evolución, pero sin rebasar los límites de sus valores normales. Esto como es obvio, se corresponde con una actividad osteoblástica aumentaday traduce indirectamente el poder inductivo de la osteogénesis de la

\section{Evolución hematológica}

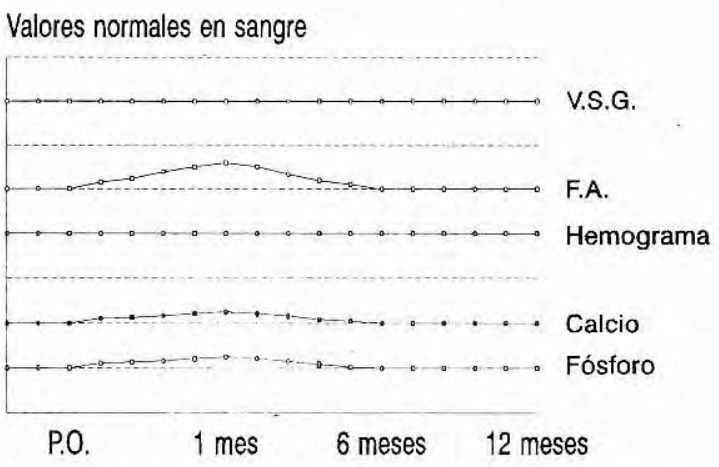

Las curvas representan la media evolutiva de cada uno d los parámetros

figura 2. Variación de los principales parámetros determinados en los análisis clínicos de los pacientes implantados.

Tabla 2. Evaluación clínica del edema y el dolor por grados y según el tiempo de evolución

\begin{tabular}{|c|c|c|c|c|c|c|}
\hline Paciente & & P.O. & 7 días & 30 días & 180 días & 1 año \\
\hline 1 & $\begin{array}{l}\text { DOLOR } \\
\text { EDEMA }\end{array}$ & $\begin{array}{l}11 \\
1\end{array}$ & $\begin{array}{l}1 \\
0 \\
\end{array}$ & $\begin{array}{l}0 \\
0 \\
\end{array}$ & $\begin{array}{l}0 \\
0 \\
\end{array}$ & $\begin{array}{l}0 \\
0 \\
\end{array}$ \\
\hline 2 & $\begin{array}{l}\text { DOLOR } \\
\text { EDEMA }\end{array}$ & $\begin{array}{l}\text { II } \\
0 \\
\end{array}$ & $\begin{array}{l}0 \\
1 \\
\end{array}$ & $\begin{array}{l}0 \\
0 \\
\end{array}$ & $\begin{array}{l}0 \\
0 \\
\end{array}$ & $\begin{array}{l}0 \\
0 \\
\end{array}$ \\
\hline 3 & $\begin{array}{l}\text { DOLOR } \\
\text { EDEMA }\end{array}$ & $\begin{array}{l}\text { II } \\
0 \\
\end{array}$ & $\begin{array}{l}\text { II } \\
0 \\
\end{array}$ & $\begin{array}{l}0 \\
0\end{array}$ & $\begin{array}{l}0 \\
0 \\
\end{array}$ & $\begin{array}{l}0 \\
0 \\
\end{array}$ \\
\hline 4 & $\begin{array}{l}\text { DOLOR } \\
\text { EDEMA } \\
\end{array}$ & $\begin{array}{l}11 \\
1\end{array}$ & $\begin{array}{l}1 \\
1\end{array}$ & $\begin{array}{l}0 \\
0 \\
\end{array}$ & $\begin{array}{l}0 \\
0 \\
\end{array}$ & $\begin{array}{l}0 \\
0 \\
\end{array}$ \\
\hline 5 & $\begin{array}{l}\text { DOLOR } \\
\text { EDEMA }\end{array}$ & $\begin{array}{l}11 \\
1\end{array}$ & $\begin{array}{l}1 \\
1\end{array}$ & $\begin{array}{l}1 \\
1 \\
\end{array}$ & $\begin{array}{l}1 \\
0 \\
\end{array}$ & $\begin{array}{l}0 \\
0 \\
\end{array}$ \\
\hline 6 & $\begin{array}{l}\text { DOLOR } \\
\text { EDEMA }\end{array}$ & $\begin{array}{l}1 \\
0 \\
\end{array}$ & II & $\begin{array}{l}0 \\
0\end{array}$ & $\begin{array}{l}0 \\
0 \\
\end{array}$ & $\begin{array}{l}0 \\
0 \\
\end{array}$ \\
\hline 7 & $\begin{array}{l}\text { DOLOR } \\
\text { EDEMA }\end{array}$ & $\begin{array}{l}11 \\
0 \\
\end{array}$ & $\begin{array}{l}1 \\
0 \\
\end{array}$ & $\begin{array}{l}0 \\
0\end{array}$ & $\begin{array}{l}0 \\
0 \\
\end{array}$ & $\begin{array}{l}0 \\
0\end{array}$ \\
\hline 8 & $\begin{array}{l}\text { DOLOR } \\
\text { EDEMA }\end{array}$ & II & $\begin{array}{l}1 \\
1\end{array}$ & $\begin{array}{l}0 \\
0 \\
\end{array}$ & $\begin{array}{l}0 \\
0 \\
\end{array}$ & $\begin{array}{l}0 \\
0 \\
\end{array}$ \\
\hline 9 & $\begin{array}{l}\text { DOLOR } \\
\text { EDEMA }\end{array}$ & II & II & $\begin{array}{l}0 \\
\text { III }\end{array}$ & $\begin{array}{l}0 \\
\text { II }\end{array}$ & $\begin{array}{l}0 \\
\text { II }\end{array}$ \\
\hline 10 & $\begin{array}{l}\text { DOLOR } \\
\text { EDEMA }\end{array}$ & $\begin{array}{l}11 \\
1\end{array}$ & $\begin{array}{l}1 \\
1\end{array}$ & $\begin{array}{l}0 \\
0\end{array}$ & $\begin{array}{l}0 \\
0\end{array}$ & $\begin{array}{l}0 \\
0\end{array}$ \\
\hline
\end{tabular}


hidroxiapatita. Al final del tratamiento el comportamiento hematológico de todos los pacientes era completamente normal.

Al evaluar las radiografías encontramos que a los 6 meses de evolución la mitad de los pacientes presentaban una imagen radiográfica compatible con una osteointegración positiva y al año de evolución ya todos tenían similar respuesta hueso-implante. Realmente no concedimos valor al tiempo, por cuanto la mayor parte de los pacientes presentaban lesiones con gran riesgo de no solución, y para nosotros primaba conseguir su curación con independencia de la rapidez con que ocurriera. Así, por ejemplo, los dos primeros pacientes de este grupo (tabla1) presentaban tumoraciones que habían sido tratadas previamente por curetaje y relleno con hueso autólogo o de banco. Dichas tumoraciones recidivaron. Según la literatura, la tasa de recidivas es de hasta un $25 \%$ en los quistes simples cuando requieren reintervención tras el curetaje, por lo que algunos autores proponen una diafisectomía subperióstica parcial en estos casos, con lo cual se consigue la reducción de la tasa de recidivas a un 5\%. El injerto óseo en general tiene una gran tendencia a la reabsorción, además de demostrar una baja capacidad osteogénica y en algunos casos elevada respuesta inmunológica (3035). Es fácil comprender, por lo tanto, nuestra satisfacción al conseguir la curación de estas lesiones recidivantes en dos de nuestros pacientes (figura 3 ).

Otro ejemplo de consideración lo constituye el sexto paciente de este grupo, una muchacha de 18 años con un condroblastoma que invadía aproximadamente el $70 \%$ de la cabeza del fémur, con vaciamiento de la epífisis hasta la región subcondral. En este caso, pensamos que el relleno con injerto autólogo o de banco no garantizaría las exigencias mecánicas de la carga de peso hasta después de unos 12 meses de evolución, además de las molestias y posibles complicaciones en ia obtención de hueso autólogo o de la magnitud del injerto a usar en el caso de hueso de banco. El condroblastoma es un tumor que debe considerarse maligno en potencia y se plantean recidivas entre un $10 \%$ y un $38 \%$ tras el curetaje o injerto óseo [36].
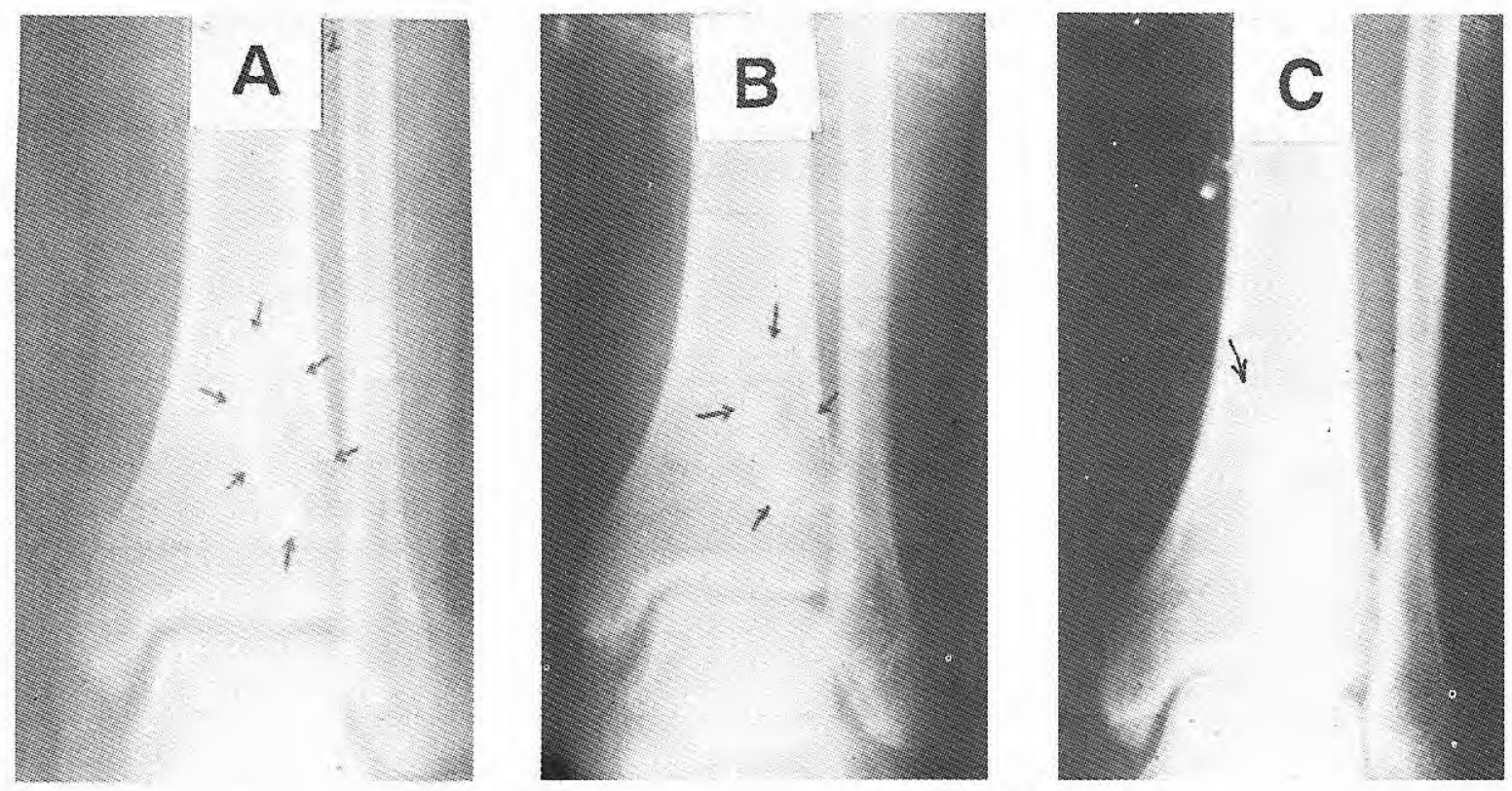

figura 3. Radiografías del paciente 2 con un defecto fibroso metafisario recidivante en la tibia izquierda, antes de la intervención (A), a los 90 días de la implantación (B) y al año de evolución (C). 
Esta paciente deambula libremente con apoyo desde los 6 meses de evolución y, en la actualidad, está clínicamente normal y en sus radiografías se observa una respuesta hueso-implante muy buena (figura 4).

El estudio comparativo inicial de la densidad relativa del implante y del hueso vecino mediante el sistema de procesamiento digital de imágenes Ortopack, muestra en general que:

- Ladensidad relativa del implante evolutivamente se asemeja a la del hueso normal.

- No se notan cambios de importancia en la densidad relativa de los tejidos vecinos.

- La densidad relativa en la unión hueso-implante es similar a la del hueso normal una vez que existe osteointegración positiva.

De acuerdo con estas experiencias, es conveniente destacar que, si bien es cierto que los biomateriales no constituyen una solución absoluta para todos los defectos óseos, sí es bien claro que entre sus bondades se cuenta la de constituir un recurso terapéutico muy útil y siempre disponible para ser usado por el cirujano ortopédico en el momento que así lo requiera y en las cantidades y condiciones de esterilidad óptimas.

Las complicaciones presentadas en todos estos casos pueden ser consideradas como ligeras y se manifestaron en sólo tres pacientes.

La primera complicación estuvo dada por osificación heterotópica en el caso de la tumoración pelviana. Es de destacar que ésto puede ocurrir en el curso de cualquier intervención quirúrgica sobre hueso, pero en este caso específico, teniendo en cuenta lo atraumático y delicado del proceder operatorio, la no desperiostización y la hemostasia adecuada, nos vemos obligados a considerar como elemento de mucha fuerza el incremento de la actividad osteogénica en la zona bajo la inducción del bioimplante.
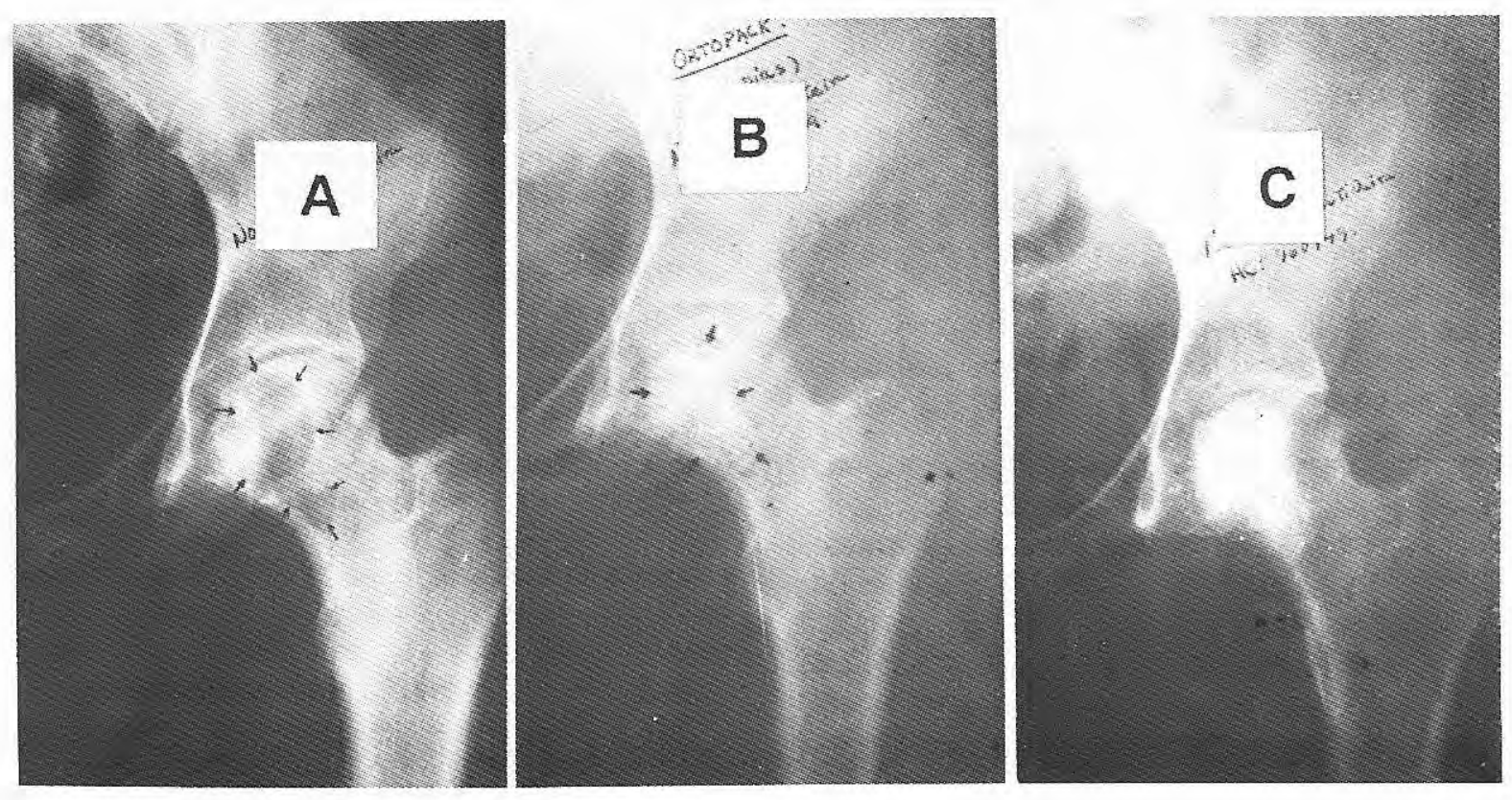

figura 4.-Radiografías del paciente 6 que presentaba un condroblastoma en el fémur izquierdo (A) y su evolución postoperatoria a los 30 días (B) y a los 180 días (C). 
La segunda complicación estuvo dada por edema persistente del miembro inferior en el noveno paciente, lo cual motivó una evaluación regular del mismo; no obstante, para nosotros, este edema no tuvo valor significativo relacionado con el implante, por cuanto no hubo signos de inflamación y además en la cirugía de las extremidades inferiores, es más la regla que la excepción, la persistencia del edema muchos meses después de la intervención.

$Y$, finalmente, la quinta paciente presentó una metatarsalgia postquirúrgica que pudimos comprobar estuvo relacionada con una apoyo inadecuado por dolor y por miedo a la carga del peso sobre la zona de operación.

\section{Conclusiones}

Al evaluar el resultado final de nuestro estudio, siguiendo los criterios de respuesta propuestos para este trabajo, encontramos nueve pacientes evaluados como excelentes y sólo uno evaluado como regular su resultado final. Esta nominación la obtuvo este paciente por mantener un edema persistente de la extremidad operada.

En cambio, al analizar el éxito o fracaso de nuestra terapéutica podemos concluir que el tratamiento empleado constituyó un éxito en el $100 \%$ de los casos, por cuanto se obtuvo la curación sin complicaciones graves y sin que existan evidencias de rechazo biológico del material usado. Todo lo anterior, corrobora y refuerza el conocimiento de la literatura mundial en relación a las bóndades de la hidroxiapatita como material implantológico de eficacia en el relleno de defectos de sustancia ósea.

La hidroxiapatita porosa coralina HAP-200 constituye un excelente biomaterial para las reconstrucciones del tejido óseo en ortopedia y traumatología. En el presente trabajo ha quedado demostrada su alta biocompatibilidad y capacidad para regenerar el tejido vivo en el tratamiento de diferentes tipos de lesiones, sin encontrarse ninguna respuesta o reacción adversa al material.

\section{Referencias}

1. Albee FH. Studies in bone growth. Triple calcium phosphate as a stimulus to osteogeneis. Ann Surg 1920; 71: 32 .
2. Levitt GE, Craytoin PH, Monroe EA, Condrate RA. Forming methods for apatite prosthesis. J Biom Mater Res 1969; 3: 683.

3. Monroe ZA, Votawa W, Bass DB, McMullen J. New calcium phosphate ceramic material for bone and tooth implants. J Dent Res 1971; 50: 860.

4. Hench LL, Splinter RJ, Allen WC, Greeniee TK. Bonding mechanism at the interface of ceramic prosthetic materials. J Biom Mater Res 1971; 2: 117.

5. Clarke WJ, Driskell TD, Hassler CR, et al. Calcium phosphate resorbable ceramics, a potenial alternative to bone grafting. IADR Progr \& abast 1973; 52: abstr 259.

6. Roy DM, Linnehan SA. Hydroxiapatite formed from coral skeleton carbonate by hydrotermic exchange. Nature $1974 ; 247: 220$

7. White RA, Weber JM, White EW. Replamineform: A new process for preparing porous ceramic, metal and poly-mer prosthetic materials. Science 1972; 176: 187.

8. Jarcho M, Salisbury RL, Thomas MB, Doremus RH. Synthesis and fabrication of tricalcium phosphate ceramic for potenial prosthetic application. J Mater Sci 1979 14: 142 .

9. Shimazaki K, Mooney V. Comparative study of porous hydroxyapatite and tricalcium phosphate as bone substitute. J Orthop Res 1985; 3:301-310.

10. Bower GM, Vargo JW, Levy B, et al. Histologic observations following the placement of tricalcium phosphate implants in human intrabony defects. JPeriodontol 1986; 57: 286-287.

11. Tracy DM, Doremus RH. Direct electron microscopy studies of the bone-hydroxiapatite interface. J Biomed Mater Res 1984; 18: 719-726.

12. Shibuya T, Takeuchi H, Ono M. Bonding behavior between two bioactive ceramics in vivo. J Biomed Mater Res 1987; 21: 1109-1123.

13. de Groot K. Ceramic of calcium phosphates: Preparations and propiertes. In Bioceramics of calcium phosphate, $\mathrm{K}$. de Groot ed, Boca Raton, FL, CRC Press 1983; 100-114.

14. González R, Guerra-López J. Materiales bioactivos para implantes óseos. Caracteristicas y aplicaciones. La Habana: Ed. CNIC., 1993.

15. Socarrás E. Evaluación clínica de hidroxiapatita porosa obtenida a partir de corales marinos. Tesis de doctorado, La Habana, 1993.

16. Nery EB, Lynch KL, Hirthe WM, Mueller KH. Bioceramic implants in surgically produced intrabony de-fects. $J$ Periodont 1975; 46: 328.

17. Jarcho M. Hydroxyapatite synthesis and cha-racterization in dense polycrystalline forms. J Mater Sci 1976; 11: 2027.

18. Jarcho M. Calcium phosphate ceramics as hard tissue prosthetic. Clin Orthopaed 1981; 157: 259. 
19. Jarcho M. Biomaterials aspects of calcium phosphates: properties and applications. Dent Clin N Am 1986; 30: 25.

20. Denissen HW, de Groot K. Immediate dental root implants from synthetic dense calcium hydroxylapatite implants. J Prosthet Dent 1979; 42: 551.

21. de Groot K, Ducheyne P. In vivo surface activity of hydroxyapatite alveolar bone substitute. J Biomed Mater Res. 1981; 15: 441.

22. de Putter C, de Groot K, Sillevis-Smitt PAE. Im-plants of dens hydroxyapatite in prosthetic dentistry. In: Clinica! applications of biomaterials. New York: J. Wiley and sons, 1982; 123-131.

23. Uchida A, Kurisaki E, Ono K. The use of bioactive ceramics for bone tumor surgery. In: Yamamuro, T.. Hench, LL. Wilson, J, eds. CRC handbook of bioactive ceramics.Volu-me II. Calcium phosphate and hydroxyapatite ceramics. Boca Raton: CRC Press: 1990: 341-344.

24. Klein CP, Dreissen AA, de Groot K. Relationship between the degradation behavior of calcium phosphate ceramics and their physical-chemical characteristics and ultraestructural geometry. Biomaterials 1984; 5: 157.

25. Klein CP, de Groot K, Dreissen AA, van der Lubbe HBM. Interaction of biodegradable B-Whitlockite ceramics with bone tissue: an in vivo study. Biomaterials 1985; 6: 189.

26. Klein CP, de Groot K, Dreissen AA, van der Lubbe HBM. A comparative study of different B-Whitlockite cera-mics in rabbit cortical bone with regard to their biodegra-dation behavior. Biomaterials 1986; 7: 144.

27. Coralina. Biomateriales para implantes óseos. La Habana: Ed. CNIC. 1992.

28. Ortopack. Sistema de procesamiento digital de imágenes.ICID- minsap. Hospital Militar Central Carlos J. Finlay. La Habana, 1991.

29. Turek S. Ortopedia. Principios y aplicaciones. Tomo 2. La Habana: Edit. Científico-Técnica, 1982; 132.

30. Campbell A. Cirugía ortopédica. Sexta Edición. ICL 1980; Tomo I: 1297.

31. Burchardt H, Busbec GA. Repair of experimental autologous grafts of cortical bone. J Bone Jt Surg 1975; 57 A: $814 ; 819$.

32. Bahn SL. A bone substitute. Oral Surg, Oral Med, Oral Path. 1966; 21: 672-681.

33. Baker RD, Terry BC. Long-term results of alveolar ridge augmentation. J Oral Surg 1970; 37: 486-489.

34. Mellonig TJ, Bowers GM. Comparison of bone graft materials. Part. II. New bone formation with autografts and allografts: an histological evaluation. J Periodontol 1981; 52: 297-302.

35. Oklund SA, Prolo DJ, et al. Quantitative comparisons of healing in cranial fresh autograft, frozen autograft, and allograft in canine skull defects. Clin Orthop 1986; 205: 269-291.

36. Campbell A. Cirugía ortopédica. Sexta Edición, ICL 1980; Tomo II; 1310. 